\title{
Broadband Quasi-Chebyshev Bandpass Filters With Multimode Stepped-Impedance Resonators (SIRs)
}

\author{
Yi-Chyun Chiou, Student Member, IEEE, Jen-Tsai Kuo, Senior Member, IEEE, and Eisenhower Cheng
}

\begin{abstract}
Planar broadband bandpass filters of order up to 9 are synthesized based on the multimode property of stepped-impedance resonators (SIRs). Based on the transmission line theory, the modal frequencies of the SIRs are calculated based on the impedance and length ratios of its hi- and low- $Z$ segments. In the synthesis, the SIR coupling schemes are determined by the split mode graphs. Using one, two, two, three, and three dual- or triple-mode SIRs, quasi-Chebyshev filters with four, six, six, eight, and nine transmission poles, respectively, are synthesized with a fractional bandwidth (BW) $\Delta=50 \%$. Emphasis is placed not only on designing the $\mathrm{I} / \mathrm{O}$ coupling structures for matching the external $Q\left(Q_{\text {ext }}\right)$ and the circuit $\mathrm{BW}$, but also on matching the resonant peaks of the circuit with the nominal Chebyshev poles. Measured results of experimental circuits show good agreement with simulated responses.
\end{abstract}

Index Terms-Bandpass filter (BPF), broadband, external $Q$, multimode, stepped-impedance resonator (SIR).

\section{INTRODUCTION}

$\mathbf{I}$ $\mathrm{N}$ THE RF front end of a modern communication system, especially for satellite and mobile applications, high-performance RF/microwave bandpass filters (BPFs) are essential devices. The multimode BPFs have many attractive features such as a simple design procedure, high selectivity, and low cost. Recently, many researches on multimode resonator filters have been published for innovative design and analysis methods [1]-[5]. In [1], a narrowband third-order Chebyshev filter is realized by a triple-mode cavity. A shorted waveguide stub is used to set up couplings between the degenerate modes. The method developed in [2] significantly extends the design possibilities for multimode cavity filters. Several degenerate resonances of a cavity are simultaneously coupled to the same I/O port. A third-order elliptic function filter is realized with one triple-mode cavity. In [3], a six-pole pseudoelliptic function BPF based on the sixfold degeneracy of a single cavity is presented. The design achieves significant savings in mass and volume when compared to six-pole dual- and/or triple-mode filters of equivalent performance. In [4], new common multimode cavity multiplexing and double-band filter methods are introduced. In [5], dual- and triple-mode resonators are used to implement miniaturized dielectric resonator filters.

Manuscript received February 11, 2006; revised April 10, 2006. This work was supported under the MoE ATU Program and by the National Science Council, Taiwan, R.O.C., under Grant NSC 94-2213-E-009-073 and Grant NSC 94-2752-E-009-003-PAE.

The authors are with the Department of Communication Engineering, National Chiao Tung University, Hsinchu, 300 Taiwan, R.O.C. (e-mail: jtkuo@ @c. nctu.edu.tw)

Digital Object Identifier 10.1109/TMTT.2006.879131
It is noted that all of above-mentioned multimode resonator filters are three-dimensional and have a relatively narrow band. They are designed based on degenerated modes, i.e., modes with identical resonant frequencies. The stepped-impedance resonators (SIRs), on the other hand, have a planar structure and are advantageous in designing BPFs due to its versatile resonant characteristics [6]-[10]. One of the key features of an SIR is that its resonant frequencies can be easily altered by tuning its geometric parameters. For example, in [6], the SIR filters are designed to have a wide stopband by locating the first higher order resonance as far away from the fundamental frequency as possible.

Recently, periodic stepped-impedance ring resonators (PSIRRs) [7] have been proposed to design dual-mode filters. The two modes are orthogonal and have the same frequencies, and perturbation is required to split up the resonances and create a narrow passband. The filters in [8]-[10], on the other hand, have a broadband characteristic by incorporating the first three SIR modes at distinct frequencies into the passband. Five-pole filters can be built up with a single triple-mode SIR and an excitation structure with strong coupling. The impedance junctions between the excitation and the SIR are capable of creating two additional transmission poles.

In this paper, we aim at developing a systematic procedure for synthesizing broadband BPFs based on single or plural multimode SIRs. When only a single SIR is involved, the design is similar to those in [8]-[10]. Each SIR is treated as a multimode cavity and contributes two or three resonances at different frequencies to the circuit. The resonances of coupled SIRs are tuned to match the transmission poles of a Chebyshev passband. The BPFs will have a quasi-Chebyshev response since the final poles deviate slightly from the designated positions due to the I/O coupling. To this end, the impedance and length ratios of the SIRs are properly chosen based on resonant spectrum in readiness. Based on the external $Q\left(Q_{\text {ext }}\right)$ formulas, proper I/O coupling is devised to meet the bandwidth (BW) specification. For demonstration, four-, six-, eight-, and nine-pole BPFs are fabricated and measured.

\section{DuAl-Mode AND TRIPLE-ModE SIRs}

The resonant frequencies of an SIR in Fig. 1 can be calculated by the following two transcendental equations [6], [11]:

$$
\begin{aligned}
& \tan \theta_{1}=R \cot \theta_{2} \text { (odd mode) } \\
& \cot \theta_{1}=-R \cot \theta_{2} \text { (even mode) }
\end{aligned}
$$

where $R=Z_{2} / Z_{1}$ is the impedance ratio and $\theta_{1}$ and $\theta_{2}$ are electrical lengths of the microstrip sections with characteristic 


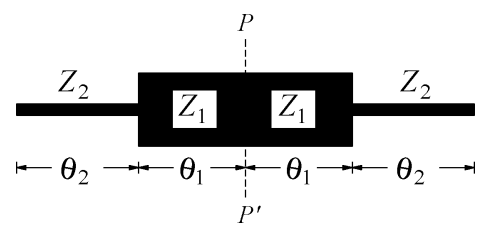

Fig. 1. Geometry of an SIR. $Z_{2}>Z_{1}$ in this study.

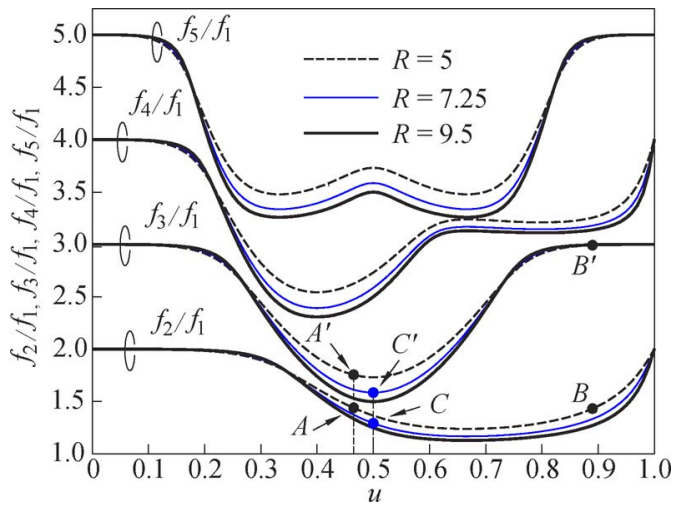

Fig. 2. Resonant spectrum of SIRs with $R=5,7.25$, and 9.5. All resonances are normalized with respect to the fundament frequency $f_{1}$. The horizontal axis $u=\theta_{2} /\left(\theta_{1}+\theta_{2}\right)$. (Color version available online at: http://ieeexplore.ieee. org.)

impedances $Z_{1}$ and $Z_{2}$, respectively. The fundamental and consecutive higher order resonances occur alternatively in the odd and even modes. Fig. 2 plots $f_{2}$ through $f_{5}$ normalized with respect to the fundamental frequency $f_{1}$ for $R=5,7.25$, and 9.5. The horizontal axis is the length ratio defined as

$$
u=\frac{\theta_{2}}{\theta_{1}+\theta_{2}} .
$$

The data shown in Fig. 2 form an important basis of our design for determining the SIR geometry. The SIRs used here are either dual- or triple-mode elements. The center frequency of a dual-mode element is defined as the arithmetic mean of the two resonances, and that of a triple-mode SIR is at its second resonance, provided that the three resonances are equally spaced. In the following demonstration for four-, six-, and nine-pole filters, either dual- or triple-mode SIRs with identical resonances are used; while for an eighth-order BPF, both dual- and triple-mode elements with distinct resonant frequencies are used.

\section{Circuit Synthesis AND MeAsurement}

The insertion loss function of a $k$ th-order Chebyshev filter can be expressed as [12]

$$
\left|S_{21}\right|^{-2}=1+\alpha^{2}\left|T_{k}(x)\right|^{2}
$$

where $T_{k}(x)$ is the $k$ th-order Chebyshev polynomial of the first kind and $\alpha$ specifies the passband ripple level. The frequencies corresponding to $|x|=0$ and 1 are center frequency and band edges, respectively. The $k$ transmission poles in (4), or the zeros of $T_{k}(x)$, are given as

$$
x_{n}=\cos \left(\frac{2 k+1-2 n}{2 k} \pi\right), \quad n=1,2, \ldots, k .
$$

For a $k$ th-order Chebyshev BPF with a fractional BW $\Delta$, the pole frequency $f_{n}$ can be easily calculated by

$$
\frac{f_{n}}{f_{o}}=1+x_{n} \times \frac{\Delta}{2}
$$

where $f_{o}$ is the center frequency. The idea of our design is to match the resonant frequencies of each BPF circuit with these transmission poles.

In a BPF, the couplings between the I/O feeder and end resonator and between adjacent resonators have to be properly designed. When the BPF has only one SIR, only the I/O coupling has to be considered. If there are two or more SIRs, the couplings among the SIRs are then chosen to match split-off resonant frequencies with the Chebyshev poles in (6). It is assumed that the I/O feeders do not shift the split-off frequencies significantly.

In this paper, all circuits are designed on a substrate with $\varepsilon_{r}=$ 2.2 and thickness $=0.508 \mathrm{~mm}$ and have $\Delta=50 \%$ with a $0.1-\mathrm{dB}$ ripple level. Before the $\mathrm{I} / \mathrm{O}$ feeders are equipped, the split-off poles are detected by a loose coupling scheme [6]. All simulation are done via IE3D [13].

\section{A. I/O Coupling}

For the broadband BPFs in [8]-[10], strong couplings are essentially required for the I/O structures. The couplings, however, seem to be lack of quantitative description. In this study, coupled-line stages are used to implement the required I/O couplings. The modal characteristic impedances of the stage $Z_{o e}$ and $Z_{o o}$ can be determined by the formulas in [14, Secs. 8.09 and 10.02] when the circuit BW is small and wide, respectively. Once $Z_{o e}$ and $Z_{o o}$ are known, the linewidth and gap size can be found. The linewidth, however, is one of geometric parameters of the SIR and has been fixed by the designated resonant frequencies. Thus, the I/O feeders need redesigning as follows. A coupled-line stage can be modeled as two quarter-wave lines with an admittance inverter in between [12]. The admittance value can be expressed in terms of the characteristic impedances of the stage

$$
J_{01} Z_{o}=\frac{Z_{o e}-Z_{o o}}{2 Z_{o}}
$$

where $Z_{o}$ is the reference port impedance. The external $Q\left(Q_{\text {ext }}\right)$ of the stage and the admittance inverter $J_{01}$ is related by

$$
Q_{\mathrm{ext}}=\frac{\pi}{2} \frac{1}{\left(J_{01} Z_{o}\right)^{2}} .
$$




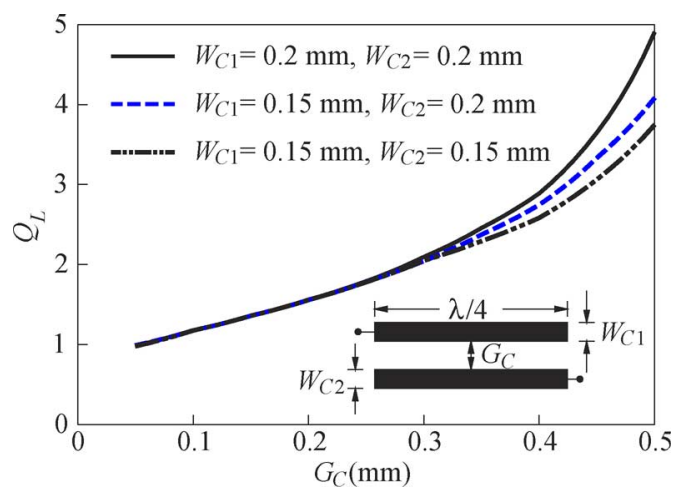

Fig. 3. Simulated loaded $Q\left(Q_{L}\right)$ for coupled-line stages versus gap size $G_{C}$. (Color version available online at: http://ieeexplore.ieee.org.)

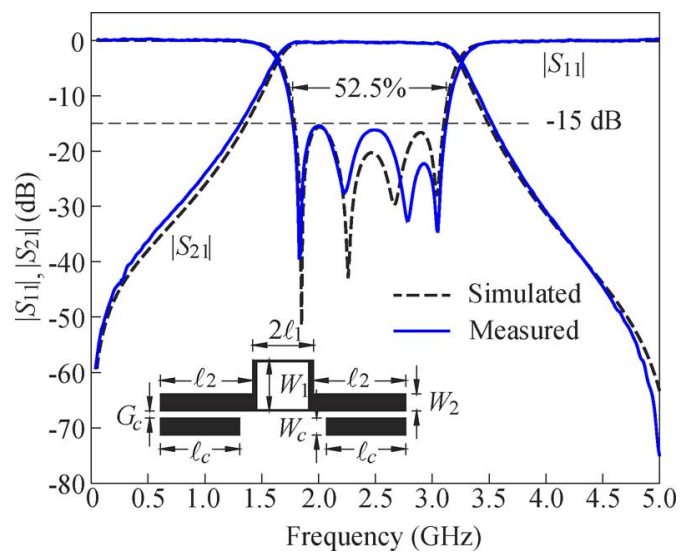

Fig. 4. Simulated and measured results of the dual-mode BPF. $W_{1}=3.76$, $W_{2}=W_{c}=0.2, \ell_{1}=3.19, \ell_{2}=26.45, \ell_{c}=22.95$, and $G_{c}=0.13($ all in millimeters). (Color version available online at: http://ieeexplore.ieee.org.)

The loaded $Q\left(Q_{L}\right)$ and the 3-dB BW $\Delta f$ of a stage is related by

$$
Q_{L}=\frac{f_{o}}{\Delta f} .
$$

Note that $\Delta f$ is specified by the stage rather than the passband response, i.e., the stage is required to have a $\Delta f$ fulfilling $Q_{\text {ext }}=Q_{L}$ for lossless cases. Fig. 3 plots the simulated $Q_{L}$ values against gap size $G_{C}$ for three sets of linewidths $W_{C 1}$ and $W_{C 2}$. It can be seen that the smaller the gap size or the linewidths, the smaller the $Q_{L}$ values. When $G_{C}<0.25 \mathrm{~mm}$, the $Q_{L}$ values of the three cases are nearly the same. In simulation, for the particular substrate with linewidth of $0.15 \sim 0.2$ $\mathrm{mm}$, the stage shows two extra transmission poles when $G_{c}<$ $0.17 \mathrm{~mm}$ and no poles when $G_{c}>0.19 \mathrm{~mm}$ [8].

\section{B. BPFs With a Single SIR}

Suppose we are designing a dual-mode filter based on two resonances at $f_{1}$ and $f_{2}$, which are designated by (5) and (6). Fig. 4 plots the simulated and measured responses of the dualmode BPF designed at $f_{o}=2.45 \mathrm{GHz}$ with $\Delta=50 \%$. The geometric parameters of the dual-mode SIR are chosen by locating $f_{1}=2.02 \mathrm{GHz}$ and $f_{2}=2.88 \mathrm{GHz}$ at the two transmission poles of $T_{2}(x)$. For $R=5$, there are two possible solutions in

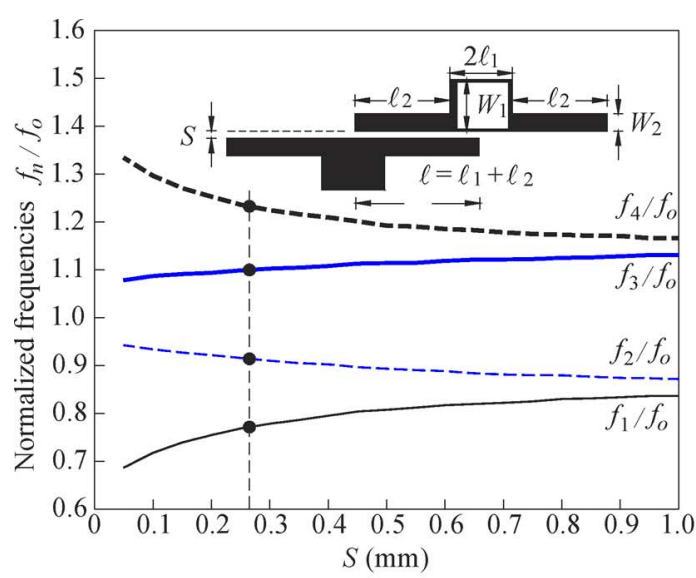

(a)

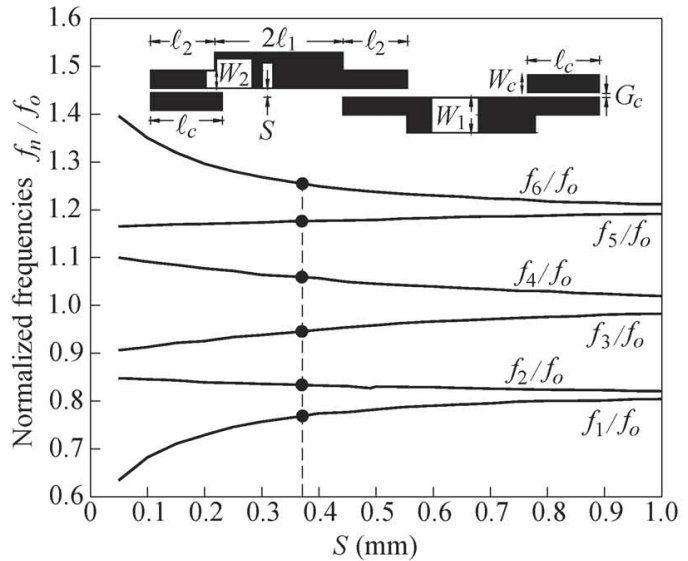

(b)

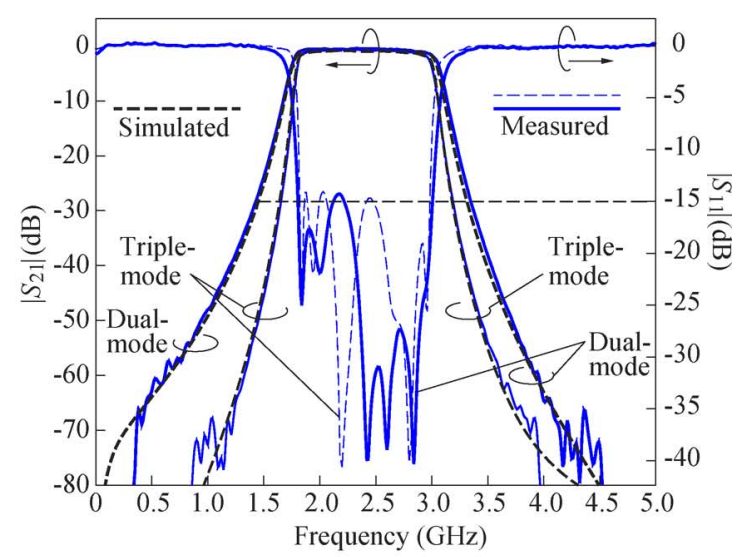

(c)

Fig. 5. Design and results of two six-pole BPFs. (a) Circuit layout and mode graph of the two coupled dual-mode SIRs. (b) Circuit layout and mode graph of the two coupled triple-mode SIRs. (c) Simulated and measured results. Dualmode BPF: $W_{1}=3.76, W_{2}=0.2, \ell_{1}=4.18, \ell_{2}=26.1, W_{c}=0.2$, $\ell_{c}=23.5, G_{c}=0.165$, and $S=0.265$. Triple-mode BPF: $W_{1}=7.24$, $W_{2}=0.15, \ell_{1}=21.5, \ell_{2}=23.8, W_{c}=0.2, \ell_{c}=24.2, G_{c}=0.215$, and $S=0.371$ (all in millimeters). (Color version available online at: http:// ieeexplore.ieee.org.)

Fig. 2, i.e., points $A$ and $B$, to have $f_{2} / f_{1}=1.426$. Obviously, $B$ is preferable to $A$ since the next resonance of the former at $B^{\prime}$ is much higher than that of the latter at $A^{\prime}$. At point $B, u=0.89$. The $\left|S_{11}\right|$ response shows that the circuit has four transmission poles. The two extra poles are from the I/O stage [8]. The length 
TABLE I

COMPARISON OF FREQUENCIES IN TRANSITION BANDS WITH SPECIFIC ATTENUATION LEVELS FOR CiRCUITS IN FIg. 5

\begin{tabular}{|c|c|c|c|c|}
\hline \multirow{2}{*}{$\begin{array}{c}\text { Frequencies } \\
(\mathrm{GHz})\end{array}$} & \multicolumn{2}{|c|}{ Theory } & \multicolumn{2}{c|}{ Measured } \\
\cline { 2 - 5 } & $k=4$ & $k=6$ & Dual-Mode & Triple-Mode \\
\hline $20 \mathrm{~dB}$ & $1.56 / 3.34$ & $1.75 / 3.15$ & $1.55 / 3.25$ & $1.70 / 3.13$ \\
\hline $30 \mathrm{~dB}$ & $1.38 / 3.52$ & $1.67 / 3.23$ & $1.39 / 3.38$ & $1.64 / 3.21$ \\
\hline $40 \mathrm{~dB}$ & $1.20 / 3.70$ & $1.57 / 3.33$ & $1.20 / 3.54$ & $1.55 / 3.31$ \\
\hline
\end{tabular}

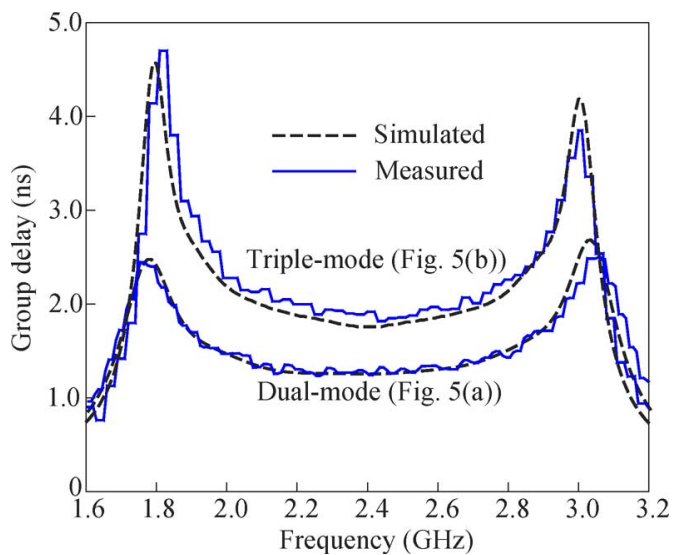

Fig. 6. Group delays for the six-pole BPFs in Fig. 5. (Color version available online at: http://ieeexplore.ieee.org.)

of the coupled-line stage $\ell_{c}=22.95 \mathrm{~mm}$ is a quarter-wavelength long at $f_{o}$. The measurement shows $\Delta=52.5 \%$ and agree well with the design. The poles at $f_{1}$ and $f_{2}$ shift to 1.85 and $3.03 \mathrm{GHz}$, respectively, after the I/O structures are applied. The shifts of these two poles are less than $9 \%$ and slightly increase the circuit $\mathrm{BW}$. The transmission zero at $5 \mathrm{GHz}$ is an inherent property of a parallel-coupled stage [15].

When the SIR is used as a triple-mode element with resonances at $f_{1}, f_{2}$, and $f_{3}$, it will be convenient to choose $u=0.5$, i.e., $\theta_{1}=\theta_{2}$, since $f_{2}$ is the arithmetic average of $f_{1}$ and $f_{3}$. It is because from (1), $\theta_{1}=\tan ^{-1} \sqrt{R}$ and $\pi-\tan ^{-1} \sqrt{R}$ at $f_{1}$ and $f_{3}$, respectively, and from (2), $\theta_{1}=\pi / 2$ at $f_{2}$, which is the midpoint of the $\theta_{1}$ values at $f_{1}$ and $f_{3}$. Points $C$ and $C^{\prime}$ in Fig. 2 show a good example. Note that this equal-space property is independent of the $R$ value. The absolute BW of a third-order circuit based on this triple-mode resonator is close to $f_{3}-f_{1}$, and it can be controlled by $R$. There have been many good BPF examples designed with a single triple-mode SIR in [8]-[10], and they will be not repeated here.

\section{BPFs With Two and Three Triple-Mode SIRs}

Two dual-mode SIRs are used to construct a six-pole BPF. The four resonant peaks of the coupled dual-mode SIRs, without the I/O stage, are symmetric about $f_{o}=2.45 \mathrm{GHz}$. Based on (6), the geometric parameters of each SIR are chosen to have two resonances at $f_{a}=\left(f_{1}+f_{2}\right) / 2$ and $f_{b}=\left(f_{3}+f_{4}\right) / 2$. Two triple-mode SIRs are also used to design a six-pole BPF with the same $f_{o}$. The width of the low- $Z$ section is $7.24 \mathrm{~mm}$ to realize $R=9.5$. The possible spurious corresponding to its transverse resonance is approximately $16 \mathrm{GHz}>6 f_{o}$.

The split mode graphs in Fig. 5(a) and (b), respectively, investigate the split off of the resonant peaks of the two dual- and two triple-mode SIRs with respect to coupling gap size $S$. The split

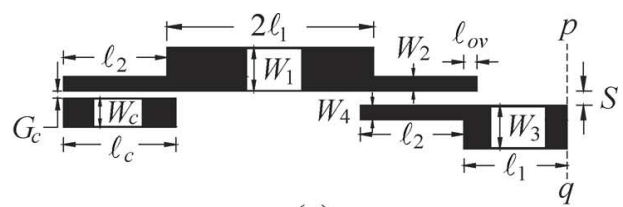

(a)

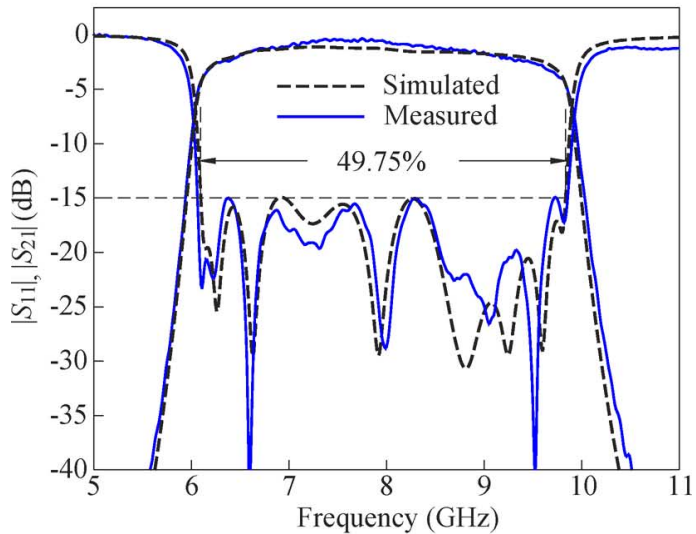

(b)

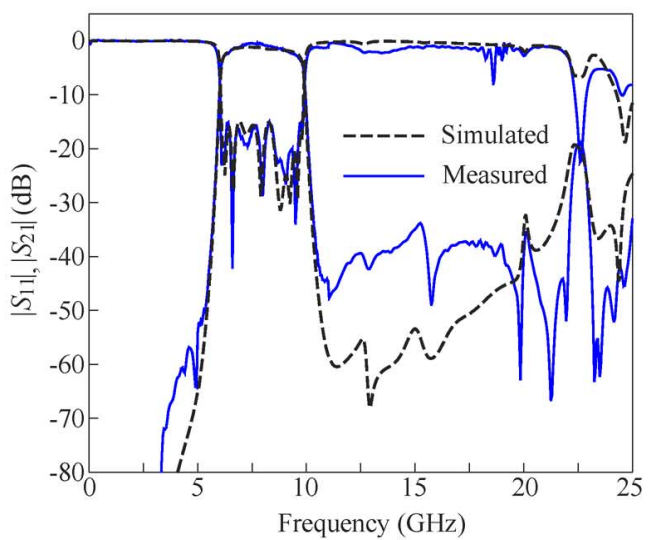

(c)

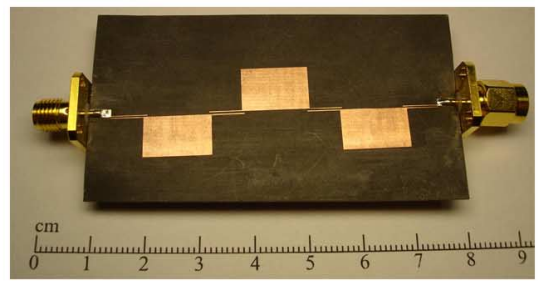

(d)

Fig. 7 Synthesis and design of a ninth-order BPF with three triple-mode SIRs (a) Half of the circuit layout. (b) Filter performance in a band from 5 to $11 \mathrm{GHz}$ (c) Simulation and measured responses in a broadband. (d) Circuit photograph. $W_{1}=7.60, W_{2}=0.17, W_{3}=8.35, W_{4}=0.15, \ell_{1}=6.29, \ell_{2}=6.11$, $\ell_{o v}=0.70, W_{c}=0.15, \ell_{c}=6.76, G_{c}=0.225$, and $S=0.375 \mathrm{~mm}$ (all in millimeters). (Color version available online at: $\mathrm{http}: / /$ ieeexplore.ieee.org.)

off increase as $S$ is decreased, as expected. $S=0.265 \mathrm{~mm}$ and $0.371 \mathrm{~mm}$ are then chosen for the dual- and triple-mode cases, respectively, to match the frequencies with the nominal poles of the fourth- and the sixth-order BPF. The maximal relative deviation of the $f_{n}$ values from those given in (6) is no larger than $1 \%$. Fig. 5(c) shows the simulated and measured results of the two BPFs. For the sake of clarity, only measured data are plotted for $S_{11}$. Measured data indicate that the in-band insertion loss is $0.9 \mathrm{~dB}$ and the return loss is $15 \mathrm{~dB}$. Note that both circuits 
TABLE II

Chebyshev Poles (Theory) and Tuned Resonant Peaks (IN GIGAHERTZ) OF THE NINTH-ORDER BPF

\begin{tabular}{|c|c|c|c|c|c|c|c|c|c|}
\hline$n$ & 1 & 2 & 3 & 4 & 5 & 6 & 7 & 8 & 9 \\
\hline Theory & 6.03 & 6.27 & 6.71 & 7.32 & 8.00 & 8.68 & 9.29 & 9.73 & 9.97 \\
\hline Tuned & 6.09 & 6.32 & 6.72 & 7.38 & 7.96 & 8.58 & 9.12 & 9.56 & 9.85 \\
\hline Meas. & 6.11 & 6.22 & 6.62 & 7.23 & 7.98 & 8.70 & 9.06 & 9.53 & 9.85 \\
\hline
\end{tabular}

have six transmission poles. Of the dual-mode BPF, again, two extra poles are resulted from the I/O stages with a gap size of $0.165 \mathrm{~mm}$. In the triple-mode case, however, there is no extra pole since gap size of the $\mathrm{I} / \mathrm{O}$ stages $G_{c}=0.215 \mathrm{~mm}$ corresponds to a relatively weak coupling.

Although both of the circuits in Fig. 5 have the same number of poles, one can easily identify that the two circuits have different attenuation rates in their transition bands by comparing the $\left|S_{21}\right|$ responses in Fig. 5(c). Table I compares the frequencies of the two circuits at specific attenuation levels with those obtained by theoretical calculations, i.e., Chebyshev function of the $k$ th-order $(k=4$ or 6$)$ with a $0.1-\mathrm{dB}$ ripple and $\Delta=50 \%$ [14]. The frequencies of the triple-mode circuit match well with those of $k=6$, while those of the dual mode agree better with those of a fourth-order Chebyshev function. This means that the two extra poles created by the I/O stages in the dual-mode BPF have little contribution to rejection levels in the transition bands.

The layouts of the circuits in Fig. 5 involve asymmetric coupled lines. Rigorous analysis for the coupling and $f_{n}$ based on the transmission line theory would be favorable for circuit design. The circuits, nevertheless, involve strong couplings and discontinuities, which need further simulation for final confirmation anyway. Thus, from the design point of view, the couplings and $f_{n}$ for the structures are directly characterized by an electromagnetic software package.

For a broadband filter, variations of group delay within the passband can be of important concern. Fig. 6 plots group delay responses for the fabricated circuits in Fig. 5. The maximum group delay variations for the dual- and triple-mode SIR circuits are 1 and $2.5 \mathrm{~ns}$, respectively. The latter shows a worse phase linearity in the passband.

Synthesis of a ninth-order BPF with three triple-mode SIRs is also studied. The circuit is designed at $f_{o}=8 \mathrm{GHz}$ and the SIRs have $R=9.6$ and $u=0.5$. Fig. 7(a) shows half of the circuit layout on the left-hand side of its plane of symmetry $p-q$. Fig. 7(b) illustrates the performance of the BPF from 5 to $11 \mathrm{GHz}$. Detailed data indicate that the measurement has in-band insertion loss $=1.3 \mathrm{~dB}$ and $\Delta=49.75 \%$ for a $15-\mathrm{dB}$ return loss. Before the $\mathrm{I} / \mathrm{O}$ coupling is imposed, the chosen circuit poles together with the Chebyshev function poles are listed in Table II. The maximal relative deviation between the theory and tuned values is only $1.8 \%$. In simulation of the entire circuit, linewidths $W_{3}$ and $W_{4}$ and the parameter $\ell_{o v}$ are finely adjusted for the in-band $\left|S_{11}\right|$ level. The poles in the measured response are also listed and show reasonably good agreement with the chosen values. Fig. 7(c) plots the simulated and measured responses up to $25 \mathrm{GHz}$. From Fig. 2, since $f_{1} \sim f_{3}$ are used for the passband, the first spurious may occur at $f_{4} \approx 2.5 f_{1}$ or $2 f_{o}$. The possible resonances in transverse direction corresponding

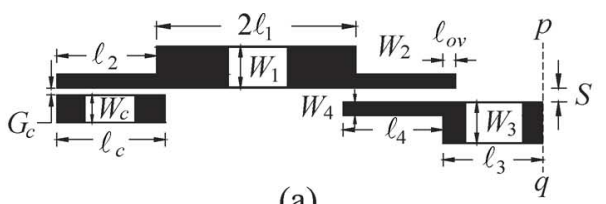

(a)

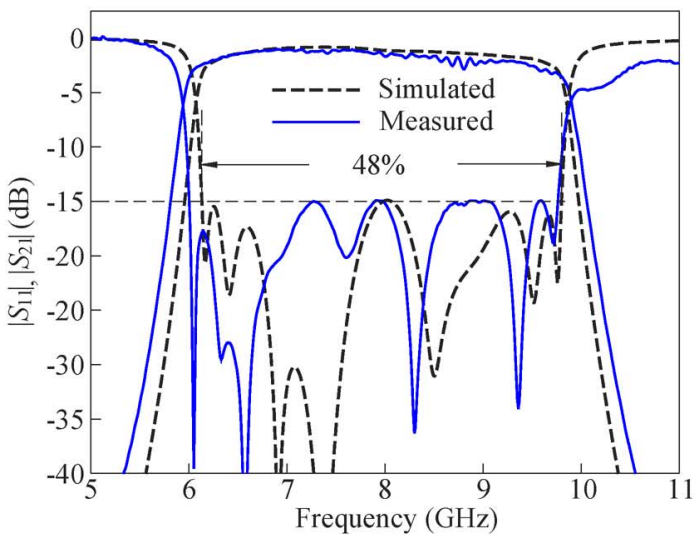

(b)

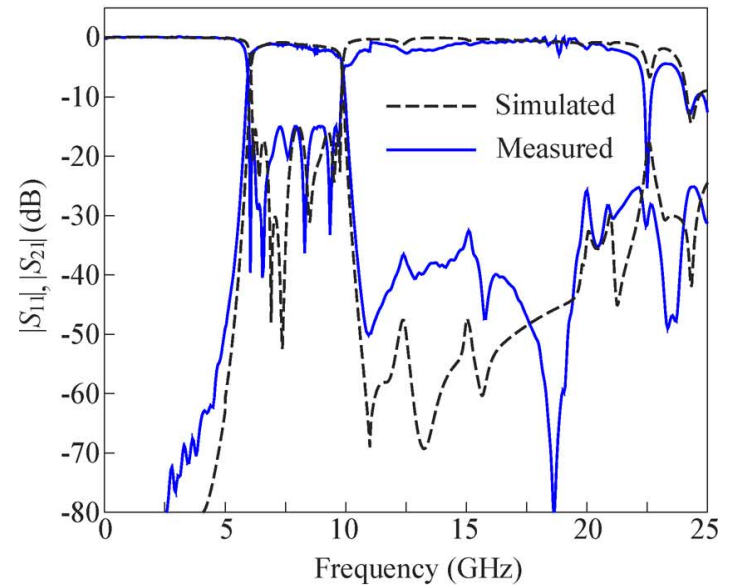

(c)

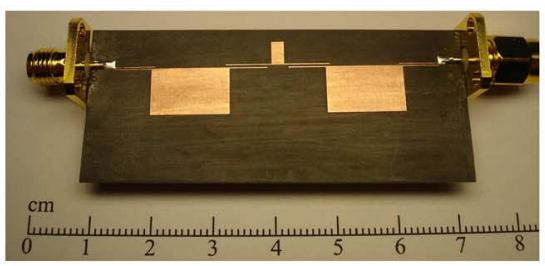

(d)

Fig. 8. Synthesis and design of an eighth-order BPF with two triple-mode and a dual-mode SIRs. (a) Layout and the split mode graph. (b) Filter performance from 5 to $11 \mathrm{GHz}$. (c) Simulation and measured responses in broadband. (d) Photograph of the circuit. $W_{1}=7.72, W_{2}=0.15, W_{3}=3.86$, $W_{4}=0.15, \ell_{1}=6.29, \ell_{2}=6.07, \ell_{3}=1.02, \ell_{4}=7.42, \ell_{o v}=0.65$, $W_{c}=0.15, \ell_{c}=6.52, G_{c}=0.22$, and $S=0.358$ (all in millimeters). (Color version available online at: http://ieeexplore.ieee.org.)

to widths of the low- $Z$ sections, $W_{1}$ and $W_{3}$, are also close to $16 \mathrm{GHz}$ or $2 f_{o}$. They are, however, suppressed by the zero created by the I/O coupled stage [14]. As a result, the 25-dB peak at $22.5 \mathrm{GHz}$ is the next spurious $f_{5} \approx 3.5 f_{1}$ or $2.8 f_{o}$ of the SIR. Fig. 7(d) shows a photograph of the fabricated circuit.

One practical important point of this study is as follows. Broadband BPFs are usually hardly realizable by the parallel-coupled configuration using the standard PCB process 
TABLE III

Chebyshev Poles (THeORY) AND TunED Resonant PEAKS (IN GIGAHERTZ) OF THE EIGHTH-ORDER BPF

\begin{tabular}{|c|c|c|c|c|c|c|c|c|}
\hline$n$ & 1 & 2 & 3 & 4 & 5 & 6 & 7 & 8 \\
\hline Theory & 6.04 & 6.34 & 6.89 & 7.61 & 8.39 & 9.11 & 9.66 & 9.96 \\
\hline Tuned & 6.17 & 6.46 & 7.00 & 7.73 & 8.35 & 8.95 & 9.50 & 9.79 \\
\hline Meas. & 6.05 & 6.33 & 6.58 & 7.62 & 8.32 & - & 9.36 & 9.72 \\
\hline
\end{tabular}

since the gap sizes are usually too small to fabricate. For example, for a ninth-order BPF with $\Delta=50 \%$ and $0.1-\mathrm{dB}$ ripple on a substrate of $\varepsilon_{r}=2.2$ and thickness $=0.508 \mathrm{~mm}$, the gap sizes range from 5 to $66 \mu \mathrm{m}$. Based on the proposed approach, however, the required gap sizes have no such difficulty, as shown in Fig. 7.

\section{BPF With Both Dual- and Triple-Mode SIRs}

Two triple-mode SIRs, with $R=10$, and one dual-mode SIR, with $R=5.75$, in the middle are organized to synthesize an eighth-order BPF. Half the circuit layout is shown in Fig. 8(a). In the mode split-off graph (not shown), the resonances $f_{3}$ and $f_{6}$ are from the dual-mode element, and they have negligible changes when $S$ is varied. The triple-mode SIRs have $f_{1}=f_{2}$, $f_{4}=f_{5}$, and $f_{7}=f_{8}$ when $S$ is large. Note that the dual- and triple-mode SIRs have identical center frequencies, but distinct individual resonances. Table III lists the theoretical pole positions and tuned resonant peaks when $S=0.358 \mathrm{~mm}$. The maximal deviation is only $2.2 \%$ and occurs at values of $f_{1}$. Fig. 8(b) shows the filter performance from 5 to $11 \mathrm{GHz}$. The measurement shows that in-band insertion loss is $1.6 \mathrm{~dB}$ and $\Delta=48 \%$ for a $15-\mathrm{dB}$ return loss. Fig. 8(c) plots the simulation and measured data up to $25 \mathrm{GHz}$. The circuit has a wide upper stopband. For example, if a $25-\mathrm{dB}$ rejection is used, the stopband extends to over $25 \mathrm{GHz}$. This could be attributed to the fact that the circuit consists of SIRs with staggered higher order resonances [16]. Fig. 8(d) presents a photograph of the circuit.

\section{CONCLUSION}

Quasi-Chebyshev BPFs of order up to 9 have been synthesized and implemented by either dual- or triple-mode SIRs. An eighth-order BPF has been realized by both of them, where they have distinct resonant frequencies. Mode graphs have been presented to depict the split off of the resonant frequencies of the coupled SIRs before the I/O feeders are applied. The resonant peaks of the multielement structure have been chosen to match the transmission poles of a Chebyshev bandpass function. It has been validated that their positions are not shifted significantly by the I/O couplings for such multielement circuits with $\Delta=50 \%$. A particular example has shown that the extra transmission poles created by the strong I/O couplings have little contributions to the rejection levels of the BPF in the transition bands. The measured responses show good agreement with the simulated results.

\section{REFERENCES}

[1] W. Steyn and P. Meyer, "A shorted waveguide-stub coupling mechanism for narrowband multimode coupled resonator filters," IEEE Trans. Microw. Theory Tech., vol. 52, no. 6, pp. 1622-1625, Jun. 2004.
[2] U. Rosenberg and W. Hagele, "Advanced multimode cavity filter design using source/load-resonance circuit cross couplings," IEEE Microw. Guided Wave Lett., vol. 2, no. 12, pp. 508-510, Dec. 1992.

[3] R. R. Bonetti and A. E. Williams, "A hexa-mode bandpass filter," in IEEE MTT-S Int. Microw. Symp. Dig., 1990, pp. 207-210.

[4] U. Rosenberg, "Multiplexing and double band filtering with commonmultimode cavities," IEEE Trans. Microw. Theory Tech., vol. 38, no. 12, pp. 1862-1871, Dec. 1990.

[5] K. Wakino, T. Nishikawa, and Y. Ishikawa, "Miniaturization technologies of dielectric resonator filters for mobile communications," IEEE Trans. Microw. Theory Tech., vol. 42, no. 7, pp. 1295-1230, Jul. 1994

[6] J.-T. Kuo and E. Shih, "Microstrip stepped impedance resonator bandpass filter with an extended optimal rejection bandwidth," IEEE Trans. Microw. Theory Tech., vol. 51, no. 5, pp. 1554-1559, May 2003.

[7] J.-T. Kuo and C.-Y. Tsai, "Periodic stepped-impedance (PSIRR) bandpass filter with a miniaturized area and desirable upper stopband characteristics," IEEE Trans. Microw. Theory Tech., vol. 54, no. 3, pp. 1107-1112, Mar. 2006.

[8] L. Zhu, H. Bu, and K. Wu, "Aperture compensation technique for innovative design of ultra-broadband microstrip bandpass filter," in IEEE MTT-S Int. Microw. Symp. Dig., 2000, pp. 315-318.

[9] W. Menzel, L. Zhu, K. Wu, and F. Bogelsack, "On the design of novel compact broadband planar filters," IEEE Trans. Microw. Theory Tech., vol. 51, no. 2, pp. 364-370, Feb. 2003.

[10] L. Zhu, C. Sun, and W. Menzel, "Ultra-wideband (UWB) bandpass filters using multiple-mode resonators," IEEE Microw. Wireless Compon. Lett., vol. 15, no. 11, pp. 796-798, Nov. 2005.

[11] M. Makimoto and S. Yamashita, "Bandpass filters using parallel coupled stripline stepped impedance resonators," IEEE Trans. Microw. Theory Tech., vol. MTT-28, no. 12, pp. 1413-1417, Dec. 1980.

[12] D. M. Pozar, Microwave Engineering, 2nd ed. New York: Wiley, 1998.

[13] IE3D Simulator. Zeland Software Inc., Fremont, CA, Jan. 1997.

[14] G. L. Matthaei, L. Young, and E. M. T. Jones, Microwave Filters, Impedance-Matching Network, and Coupling Structures. Norwood, MA: Artech House, 1980.

[15] J.-T. Kuo, S.-P. Chen, and M. Jiang, "Parallel-coupled microstrip filters with over-coupled end stages for suppression of spurious responses," IEEE Microw. Wireless Compon. Lett., vol. 13, no. 10, pp. 440-442, Oct. 2003.

[16] C.-F. Chen, T.-Y. Huang, and R.-B. Wu, "Design of microstrip bandpass filters with multiorder spurious-mode suppression," IEEE Trans. Microw. Theory Tech., vol. 53, no. 12, pp. 3788-3793, Dec. 2005.

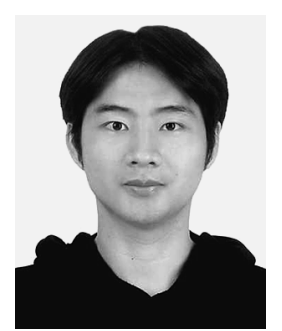

Yi-Chyun Chiou (S'04) was born in Taoyuan, Taiwan, R.O.C., on September 25, 1979. He received the B.S. and M.S. degrees in the electronic engineering from Feng Chia University (FCU), Taichung, Taiwan, R.O.C., in 2001 and 2003, respectively, and is currently working toward the Ph.D. degree in communication engineering at National Chiao Tung University (NCTU), Hsinchu, Taiwan, R.O.C.

From 2003 to 2004, he was a Lecturer with the Department of Electronic Engineering, Nan-Kai Institute of Technology, Nanto, Taiwan, R.O.C. His research interests include the design of microwave devices and associated RF modules for microwave and millimeter-wave applications.

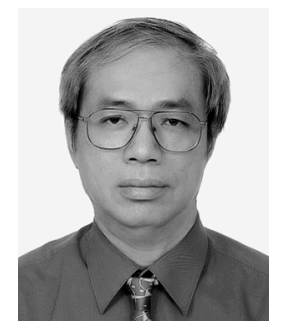

Jen-Tsai Kuo (S'88-M'92-SM'04) received the $\mathrm{Ph} . \mathrm{D}$. degree from the Institute of Electronics, National Chiao Tung University (NCTU), Hsinchu, Taiwan, R.O.C., in 1992.

Since 1984, he was with the Department of Communication Engineering, NCTU, where he is currently a Professor. From 1995 to 1996, he was a Visiting Scholar with the Electrical Engineering Department, University of California at Los Angeles (UCLA). From Aug. 2001 to July 2003, he was the Deputy Department Chair, from Aug. 2003 to July 2005, he was the Director of the Degree Program, and from February 2005 to March 2006, he was the Director of the Industrial Degree Program of the Electrical Engineering and Computer Science Colleges. His research interests include analysis and design of microwave integrated circuits (MICs) and numerical techniques in electromagnetics. 
Dr. Kuo is currently an Editorial Board member of the IEEE TRANSACTIONS ON MicrowaVe THEORY AND TECHNiQues and the IEEE MicrowaVe AND WiRELESS COMPONENTS LETTERS. He was a corecipient of the Best Paper Award of the 2002 National Telecommunication Conference, Taiwan, R.O.C.

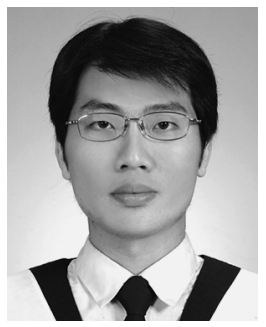

Eisenhower Cheng was born in Hualien, Taiwan, R.O.C., on January 16, 1981. He received the B.S. degree in the physics from National Tsing Hua University (NTHU), Hsinchu, Taiwan, R.O.C., in 2004, and the M.S. degree in communication engineering from National Chiao Tung University (NCTU), Hsinchu, Taiwan, R.O.C., in 2006.

His research interests include the design of microwave planar filters and RF modules for microwave and millimeter-wave applications. 